This item was submitted to Loughborough's Research Repository by the author.

Items in Figshare are protected by copyright, with all rights reserved, unless otherwise indicated.

\title{
Elastic wave dispersion in microstructured membranes
}

PLEASE CITE THE PUBLISHED VERSION

http://dx.doi.org/10.1098/rspa.2009.0516

\section{PUBLISHER}

Royal Society Publishing (@ the authors)

VERSION

AM (Accepted Manuscript)

\section{LICENCE}

CC BY-NC-ND 4.0

\section{REPOSITORY RECORD}

Lombardo, Mariateresa, and Harm Askes. 2019. "Elastic Wave Dispersion in Microstructured Membranes". figshare. https://hdl.handle.net/2134/11454. 
This item was submitted to Loughborough's Institutional Repository (https://dspace.lboro.ac.uk/) by the author and is made available under the following Creative Commons Licence conditions.

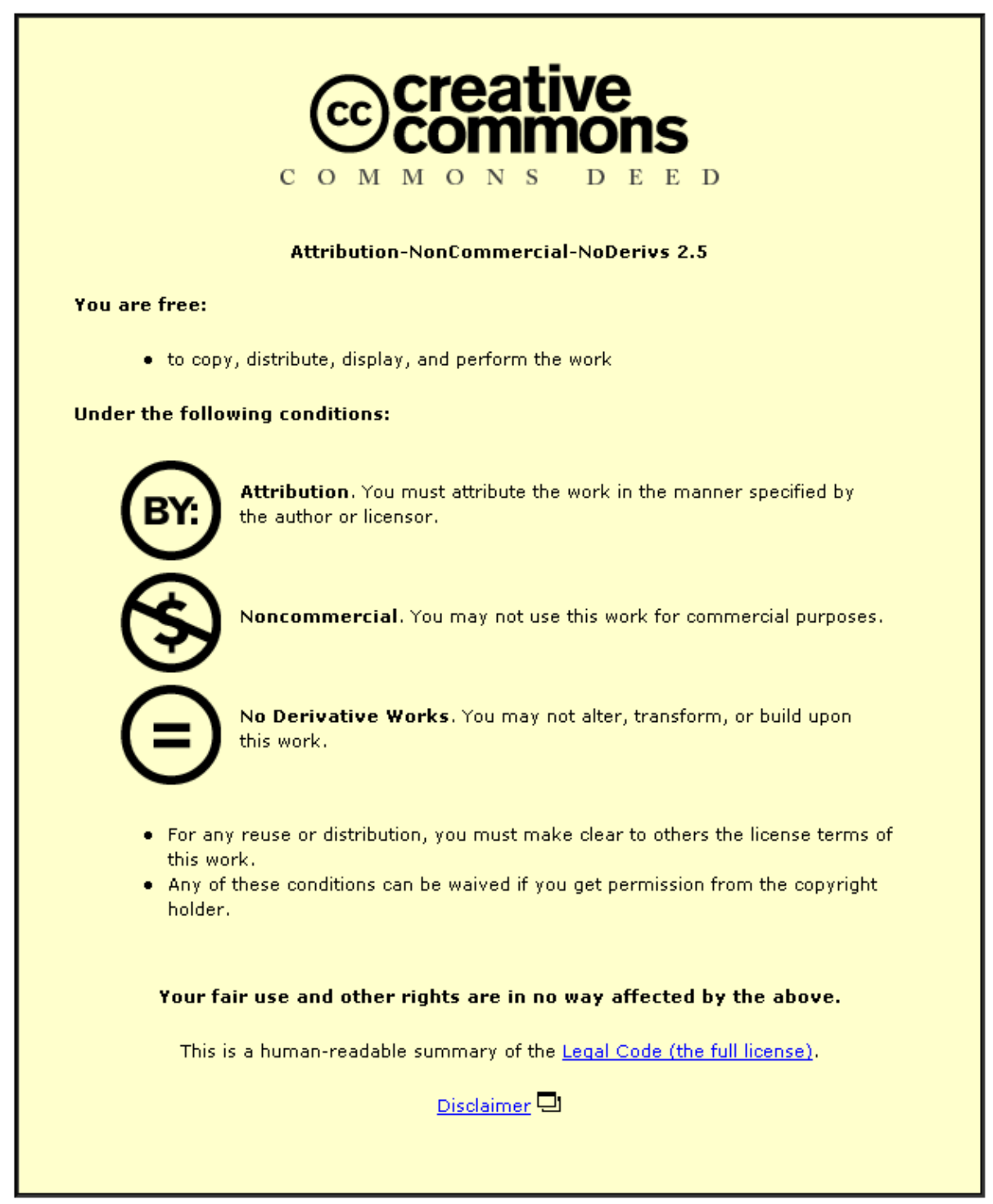

For the full text of this licence, please go to: http://creativecommons.org/licenses/by-nc-nd/2.5/ 


\title{
Elastic wave dispersion in microstructured membranes
}

\author{
By Mariateresa Lombardo and Harm Askes \\ Department of Civil and Structural Engineering, University of Sheffield, Mappin \\ Street, Sheffield S1 3JD, UK
}

The effect of microstructural properties on the wave dispersion in linear elastic membranes is addressed in this paper. The periodic spring-mass lattice at the lower level of observation is continualised and a gradient-enriched membrane model is obtained to account for the characteristic microstructural length scale of the material. In the first part of this study, analytical investigations show that the proposed model is able to capture correctly the physical phenomena of wave dispersion in microstructured membrane which is overlooked by classical continuum theories. In the second part, a finite element discretisation of microstructured membrane is formulated by introducing the pertinent inertia and stiffness terms. Importantly, the proposed modifications do not increase the size of the problem with respect to the classical elasticity. Numerical simulations are included for validation purposes. The results confirm that the structural characteristics of the material can have a huge impact on the vibrational properties, particularly in the high-frequency range.

Keywords: continualisation; higher-order continuum; lattice model; microstructure; Padé approximation; wave dispersion

\section{Introduction}

A tensioned membrane can be considered as the simplest example of a two- dimensional vibrating system, although also more complex structures may be regarded as membranes, whose fundamental characteristic is the incapability of conveying moments or shear forces. Currently, innovative practical applications exist for these structural models such as antennas and solar sails for space applications, biotechnological prostheses, and lightweight roofs designed in architecture and structural engineering (Jenkins \& Korde 2006). The use of composite materials for these high performance systems is becoming fundamental, and the request of specific properties has increased the design of fabric materials providing the flexibility and lightweight characteristics appropriate for membrane structures. Composite membranes possess a relevant microscopic structure that often influences significantly the corresponding macroscopic response. Consequently, accurate modelling of such structures and materials requires that the microstructure is accounted for.

In mechanical problems involving materials with microstructure, the existence of microscopic internal characteristic lengths, whose origin may be the lattice period for a discrete system or the inclusion size for an inhomogeneous material, introduces a size dependence into the structural response on the macroscopic level. These effects may be accurately analyzed including each individual microstructural 
element in a discrete model. However, this approach leads to a considerable computational effort because the number of degrees of freedom is normally very large. The strategy of replacing such a discrete model with an equivalent continuum, characterizing the average mechanical behavior of the actual discrete or inhomogeneous material, offers a efficient alternative since the continuum mechanics approach is computationally less expensive.

Although classical continuum theories are adequate for many static situations, their validity is limited for problems involving dynamic analysis in the high-frequency regime, wherein microstructural effects are more significant. The dispersion of waves propagating through a heterogeneous or discrete medium is extensively documented by experimentalists, and this cannot be adequately simulated using standard continuum material models (Erofeyev 2003, Jakata \& Every 2008). Dispersion is the phenomenon that waves with different wave lengths propagate with different velocities. Because a propagating wave is assumed as a superposition of harmonic components, its shape is altered when the components have different wave speeds. Waves that propagate through a classical medium are not dispersive, i.e. the wave speed is constant, independent of wave length, and waves travelling through this medium maintain a constant shape.

Enhanced continuum models constitute a class of continua accounting for the intrinsic length of microstructural details in the macrostructural material model, normally by means of additional (temporal or spatial) derivatives of the relevant state variables. It was shown that the wave dispersion in dynamics can be realistically restored by the addition of higher-order inertia terms and higher-order stiffness terms in the governing equations, see for instance the recent overview in Papargyri-Beskou et al. (2009). To this end, continualisation or homogenization strategies were proposed to account for the heterogeneous or discrete nature of materials. Following the philosophy of homogenization theory, the cases of monodimensional systems have been discussed in the works of Chen \& Fish (2001) and Fish et al. (2002) with the aim to obtain dispersive continuum models for periodic heterogeneous media. If the material at the lower scale can be assumed to be a discrete medium, continualisation techniques can be used to translate the discrete nature into a gradient-enriched continuum with the aim to preserve the essential link between the degrees of freedom of the actual model and those of the continuum (see, for instance, Metrikine \& Askes 2002). Investigations regarding the dispersion properties of granular material, whose discreteness can be recognized at the observation level, can be found in Chang \& Gao (1995), Mühlhaus \& Oka (1996), Suiker et al. (2001) and Suiker \& de Borst (2005), although care must be taken that the resulting continuum model is stable (Metrikine \& Askes 2002).

The aim of this contribution is to validate the accuracy of enhanced continuum analysis of a membrane with microstructure subject to dynamic loading. The effectiveness in the high-frequency range is investigated by means of dispersion relations. The derivation of a membrane continuum model has been already discussed for nonlinear and linear dynamics of lattices by Rosenau (1987) and Andrianov \& Awrejcewicz (2008). However, for reason of clarity the main steps in the derivation procedure will be recapped in $\S 2$. With the aim to better understand the limits of validity of the higher-order continua in the context of dynamic problems, the analysis of the dispersion properties of transverse waves propagating along different direction is presented in $\S 3$. The spatial discretization with finite elements and 
the time integration of the field equations are detailed in $\S 4$. Finally, numerical analyses of a microstructured membrane are treated in $\S 5$ with the aim to verify that the enhanced continuum models provide more realistic results than the classical continuum theory.

\section{Transverse vibrations of a two-dimensional lattice and continualization methods}

In this section the continualisation approach is employed for the construction of continuum models from associated periodic structures. The microstructured membrane is modelled by a regular lattice and taking advantage of the periodicity of the structure, the equation of motion for an inner cell is derived. Then, truncated Taylor series expansions are used to approximate the difference equations by differential equations. In this manner, the microstructural effects are accounted for by means of higher-order gradients naturally appearing in the continuum equations.

The equations governing the transverse vibrations of a two-dimensional lattice have been derived, for reasons of simplicity, assuming the hypotheses of linear elasticity. The equation of motion obtained via the standard continualisation approach is presented, and it is shown that reduces to standard elastic continuum when we account for only the first terms in the series whereas the higher-order terms increase the order of the partial differential equations. It follows that supplementary boundary conditions are required to define a well-posed problem. In order to avoid this inconvenience, the Padé approximants are introduced yielding a more effective continuum model; incidentally Padé approximants also help in overcoming instabilities as explained in $\S 3$.

\section{(a) Discrete model}

The two-dimensional spring-mass lattice that will be analyzed consists of a repeating arrangement of $N_{1} \times N_{2}$ identical particles of mass $M$, connected by springs. The interparticle distances are denoted by $\ell_{x}$ and $\ell_{y}$, in $x$ and $y$ directions respectively. As outlined in Rosenau (1987), due to the spatial periodicity of the system, the dynamics of such structures can be studied by considering only a unit cell as depicted in figure 1. If the lattice experiences small displacements in transverse direction (i.e. $z$ ) compared to its initial position in the $x-y$ plane, the membrane vibrations can be studied in the linear regime (Andrianov \& Awrejcewicz 2008).

The equation of motion governing the free transverse vibrations of an element located in the $\mathrm{m}$ th row and $\mathrm{n}$ th column is obtained by balance of forces in vertical direction as

$$
\begin{gathered}
M \frac{\partial^{2} u_{m, n}}{\partial t^{2}}=\left[\left(F_{m+1, n}-F_{m-1, n}\right)+\left(F_{m, n+1}-F_{m, n-1}\right)\right] \\
m=0,1, \ldots, N_{1} ; \quad n=0,1, \ldots, N_{2}
\end{gathered}
$$

where $u_{m, n}=u_{m, n}(t)=u\left(x_{m}, y_{n} ; t\right)$ is the transverse displacement of the mass and $F_{i, j}$ is the recall force of the stretched springs connecting the nearest-neighbour particles.

For small displacements, we may assume that the contribution of $F_{i, j}$ at all points in the lattice remains close to its equilibrium value and each contribution 


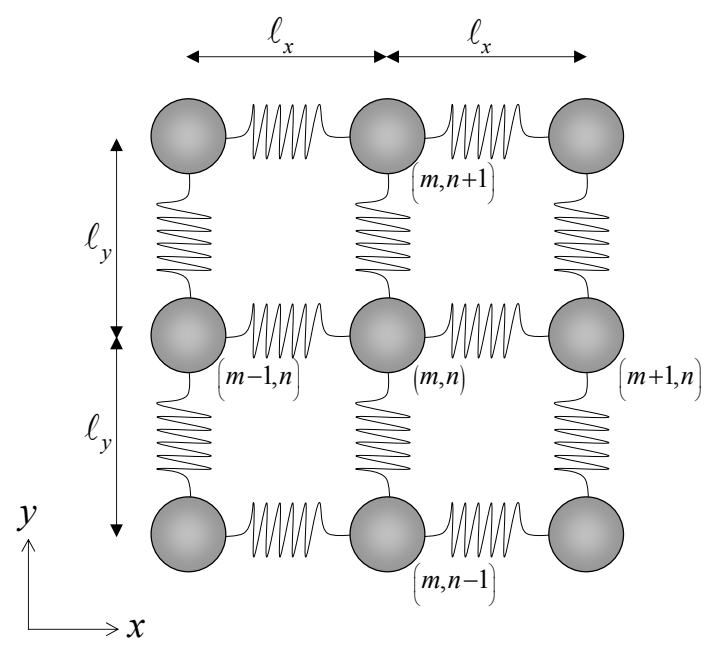

Figure 1. Two-dimensional discrete lattice in the $(x, y)$ plane.

has the form

$$
\begin{aligned}
& F_{m+1, n}-F_{m-1, n}=\frac{F_{x}}{\ell_{x}}\left[\left(u_{m+1, n}-u_{m, n}\right)-\left(u_{m, n}-u_{m-1, n}\right)\right] \\
& F_{m, n+1}-F_{m, n-1}=\frac{F_{y}}{\ell_{y}}\left[\left(u_{m, n+1}-u_{m, n}\right)-\left(u_{m, n}-u_{m, n-1}\right)\right]
\end{aligned}
$$

where now $F_{i}$ is the force of the spring in direction $i$.

For the sake of simplicity we employ a square lattice, so that $F_{x}=F_{y}$ and $\ell_{x}=\ell_{y}$, and inserting equations (2.2) into equation (2.1) the equation of motion reads

$$
M \frac{\partial^{2} u_{m, n}}{\partial t^{2}}=T\left[u_{m+1, n}-2 u_{m, n}+u_{m-1, n}\right]+T\left[u_{m, n+1}-2 u_{m, n}+u_{m, n-1}\right]
$$

where $T=\frac{F}{\ell}$ is an in-plane tensile load. The following boundary and initial conditions are applied

$$
\begin{array}{r}
u_{0, n}=u_{N_{1}, m}=u_{m, 0}=u_{m, N_{2}}=0 \\
u_{m, n}(t)=\frac{\partial}{\partial t} u_{m, n}(t)=0
\end{array}
$$

To continualize the equation (2.3), the simplest strategy suggests to replace the discrete degrees of freedom by a continuous variable field.

\section{(b) Classical continuum approximation}

Following the hypothesis of a dense lattice, a continuum approximation involves introducing a continuum displacement field $u_{m, n} \equiv u(x, y ; t)$, whereas the displacements of the neighbouring particles $u_{m \pm 1, n}$ and $u_{m, n \pm 1}$ are replaced by $u(x \pm \ell, y)$ 
and $u(x, y \pm \ell)$, respectively. Using Taylor series expansions the displacements of the neighbouring particles can be approximated according to

$$
\begin{aligned}
& u_{m \pm 1, n}=u(x \pm \ell, y ; t)=u \pm \ell \frac{\partial u}{\partial x}+\frac{\ell^{2}}{2} \frac{\partial^{2} u}{\partial x^{2}}+\ldots \\
& u_{m, n \pm 1}=u(x, y \pm \ell ; t)=u \pm \ell \frac{\partial u}{\partial y}+\frac{\ell^{2}}{2} \frac{\partial^{2} u}{\partial y^{2}}+\ldots
\end{aligned}
$$

For notational simplicity, the dependence of $u$ on the spatial coordinates and on the time $t$ is not explicitly shown. The accuracy of the derived continuum depends on the number of terms included in the Taylor series. Taking into account the terms up to the second derivatives and introducing in equation (2.3) the continuum field variables, the membrane-like continuum approximation is obtained

$$
\rho \frac{\partial^{2} u}{\partial t^{2}}=T\left[\frac{\partial^{2} u}{\partial x^{2}}+\frac{\partial^{2} u}{\partial y^{2}}\right]
$$

where $\rho=\frac{M}{\ell^{2}}$ is the mass density. If the uniform tension $T$ is sufficiently large, the transverse displacement is small (so that $T$ does not change significantly). Equation (2.6) represents the classical equation of transverse motion for a vibrating membrane uniformly stretched, bounded by a surface $\Gamma$, with initial conditions $u_{0}$ and $\frac{\partial u_{0}}{\partial t}$, and boundary conditions fixed along a portion of the edge $\Gamma_{D}$, i.e. $u=0$ on $\Gamma_{D}$ and free along the boundary $\Gamma_{N}$, i.e. $T \frac{\partial u}{\partial t}=0$ on $\Gamma_{N}$. The applicability of classical continuum models in dynamics is, however, restricted to a limited range of frequencies as will be highlighted in $\S 3$ dedicated to the dispersion analysis.

\section{(c) The quasi-continuum method and Padé approximation}

Although the application of a model with derivative of more than second order allow us to include the effect of microstructure via the parameter $\ell$ in the continuum model, the higher order of the resulting differential equation complicates the solution of continuous problems. An alternative continualisation procedure can be realised using the quasi-continuum approximations. This technique consists in the reduction of the differential-difference equation (2.3) to a partial differential equation. The continuum model is not obtained by simply replacing the difference operator in equation (2.3) by the analogous differential operator. In the first stage, the backward and forward shifts operators are introduced and for the displacements of adjacent masses the following symbolic notations hold

$$
\begin{aligned}
& u_{m \pm 1, n}=\left[\exp \left( \pm \ell \frac{\partial}{\partial x}\right)\right] u_{m, n} \\
& u_{m, n \pm 1}=\left[\exp \left( \pm \ell \frac{\partial}{\partial y}\right)\right] u_{m, n}
\end{aligned}
$$

By means of equations $(2.7 \mathrm{a})$ and $(2.7 \mathrm{~b})$ and the relation $u_{m, n}=u$, the discrete equation (2.3) is converted into a pseudo-differential equation as follows

$$
\begin{aligned}
M \frac{\partial^{2} u}{\partial t^{2}} & =T\left[\exp \left(\ell \frac{\partial}{\partial x}\right)-2+\exp \left(-\ell \frac{\partial}{\partial x}\right)\right] u \\
& +T\left[\exp \left(\ell \frac{\partial}{\partial y}\right)-2+\exp \left(-\ell \frac{\partial}{\partial y}\right)\right] u
\end{aligned}
$$


The differential operators in square brackets can be developed into Taylor series in the neighbourhood of zero (McLaurin series)

$$
\begin{aligned}
& \exp \left(\ell \frac{\partial}{\partial x}\right)-2+\exp \left(-\ell \frac{\partial}{\partial x}\right)=\ell^{2} \frac{\partial^{2}}{\partial x^{2}}+\frac{\ell^{4}}{12} \frac{\partial^{4}}{\partial x^{4}}+\frac{\ell^{6}}{360} \frac{\partial^{6}}{\partial x^{6}}+\ldots \\
& \exp \left(\ell \frac{\partial}{\partial y}\right)-2+\exp \left(-\ell \frac{\partial}{\partial y}\right)=\ell^{2} \frac{\partial^{2}}{\partial y^{2}}+\frac{\ell^{4}}{12} \frac{\partial^{4}}{\partial y^{4}}+\frac{\ell^{6}}{360} \frac{\partial^{6}}{\partial y^{6}}+\ldots
\end{aligned}
$$

Taking into account only the first term in the expansions (2.9), one obtains again the classical continuum approximation given in equation (2.6). Furthermore, the accuracy of the approximation can be increased keeping the next terms in the series, and the higher order approximation reads

$$
M \frac{\partial^{2} u}{\partial t^{2}}=T\left[\ell^{2} \nabla^{2} u+\frac{\ell^{4}}{12}\left(\frac{\partial^{4} u}{\partial x^{4}}+\frac{\partial^{4} u}{\partial y^{4}}\right)\right]
$$

where $\nabla^{2}=\frac{\partial^{2}}{\partial x^{2}}+\frac{\partial^{2}}{\partial y^{2}}$.

An alternative continuum model has been developed by using the one-point Padé approximation of the differential operator appearing on the right hand side of equation (2.10). For more details regarding the Padé approximants we refer the reader to the works by Rosenau (1986), Wattis (2000), Kevrekidis et al. (2002) and Andrianov \& Awrejcewicz (2005). Taking into account only two terms in the expansion, the operator in square brackets can be developed by means of the Padé approximation

$$
\ell^{2} \nabla^{2}+\frac{\ell^{4}}{12}\left(\frac{\partial^{4}}{\partial x^{4}}+\frac{\partial^{4}}{\partial y^{4}}\right) \approx \frac{\ell^{2} \nabla^{2}-\frac{\ell^{4}}{6} \frac{\partial^{4}}{\partial x^{2} \partial y^{2}}}{1-\frac{\ell^{2}}{12} \nabla^{2}}
$$

which leads to the following continuum model

$$
\rho\left(1-\frac{\ell^{2}}{12} \nabla^{2}\right) \frac{\partial^{2} u}{\partial t^{2}}=T\left[\nabla^{2} u-\frac{\ell^{2}}{6} \frac{\partial^{4} u}{\partial x^{2} \partial y^{2}}\right]
$$

The characteristic length $\ell$ is carried by the adjunctive inertia term on the left hand side and by the stiffness contribute on the right hand side. Also note that the introduction of the higher-order inertia and stiffness terms leads to a continuum model able to represent the wave dispersion in the microstructured material. A more detailed explanation of this issue will be given in the subsequent section devoted to the dispersion analysis.

\section{Dispersion analysis}

The dispersive character of plane waves due to inherent material characteristic length is consistent with experimental observation. Specifically, this effect becomes significant when the wavelength of the physical phenomena of interest decreases to the order of the internal length, as, for example, in problems related to shock waves. The regularization procedures introduced in the previous section are developed with 
the aim to define a phenomenological nonlocal continuum which models dispersion phenomena by capturing the characteristics of the microstructure. The effectiveness of the enhanced continuum models to represent the wave propagation characteristics of a lattice system can be evaluated by analysis of the dispersion curves. The aim of this section is to carry out a comparative study of the dispersion properties for the enhanced continuum models presented in the previous section.

\section{(a) Dispersion relations for the lattice model and the classical continuum approximation}

In the analysis of plane waves propagation in two-dimensional periodic discrete structures, a dispersion equation can be obtained by substituting into equation (2.3) a harmonic wave of the form

$$
u_{m, n}=A \exp \left[\mathrm{i}\left(\omega t-k_{x} x_{m}-k_{y} y_{n}\right)\right]
$$

where $\omega$ is the angular frequency, $A$ is the amplitude, $\mathrm{i}=\sqrt{-1}$ is the imaginary unit and $x_{m}=m \ell$ and $y_{n}=n \ell$ are the spatial coordinates, respectively. In a twodimensional model, transverse waves travel along an arbitrary direction that is at an angle $\theta$, which represents the angle between the wavevector $\boldsymbol{k}$ and the $x$ axis. In equation (3.1) $k_{x}=k \cos \theta$ and $k_{y}=\sin \theta$ define the components of the wave number in the $x$ and $y$ direction, respectively. The dispersion relation is a function $\omega=f\left(k_{x}, k_{y}\right)$ which contains information of the wave propagation characteristics of the considered material and, from the equation of motion (2.3), for the discrete lattice model is obtained as follows

$$
\widetilde{\omega}= \pm \sqrt{4 \sin ^{2}\left(\frac{k_{x} \ell}{2}\right)+4 \sin ^{2}\left(\frac{k_{y} \ell}{2}\right)}
$$

where $\widetilde{\omega}=\omega \sqrt{\frac{T}{\rho \ell^{2}}}$ is a non-dimensional frequency parameter. In the analysis of wave dispersion the non-dimensional wave numbers $k_{x} \ell$ and $k_{y} \ell$ are varied to investigate the frequencies at which waves propagate and the direction of the body wave propagation.

The dispersion relation expressed in equation (3.2) is evidently periodic in the interval $k_{x} \ell, k_{y} \ell \in[-\pi, \pi]$ and it is also symmetric with respect to the axes $k_{x} \ell=0$ and $k_{y} \ell=0$. These properties allow us to restrict the analysis to the range $k_{x} \ell, k_{y} \ell \in[0, \pi]$ that reflects the so called first Brillouin zone. A particularly convenient representation of the dispersion relations employs iso-frequency curves whose graphical interpretation provides information on the dispersion properties. The curves determined by equation (3.2) are represented in normalized form $\left(k_{x} \ell-k_{y} \ell\right.$ plane for fixed values of $\left.\widetilde{\omega}\right)$ in figure $2(\mathrm{a})$.

The wave group velocity $c_{g}$ for undamped structures is the velocity of propagation of vibrational energy, and may be expressed in the form

$$
c_{g}=\frac{\mathrm{d} \omega}{\mathrm{d} \boldsymbol{k}}=\frac{\partial \omega}{\partial k_{x}} \boldsymbol{i}+\frac{\partial \omega}{\partial k_{y}} \boldsymbol{j}
$$

where $\boldsymbol{i}$ and $\boldsymbol{j}$ are the unit vectors in the $x$ and $y$ directions, respectively. It should be noted that the group velocity is normal to the curves in the $k_{x}-k_{y}$ plane for 


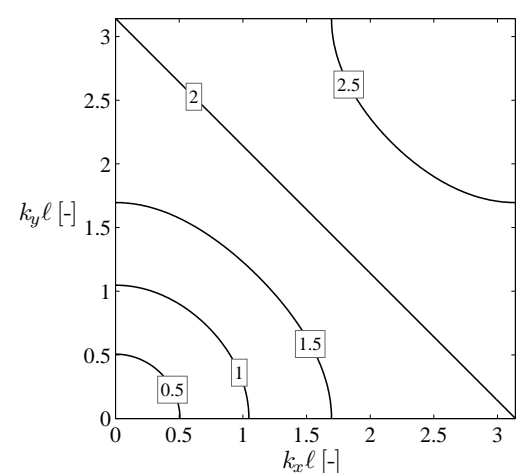

(a)

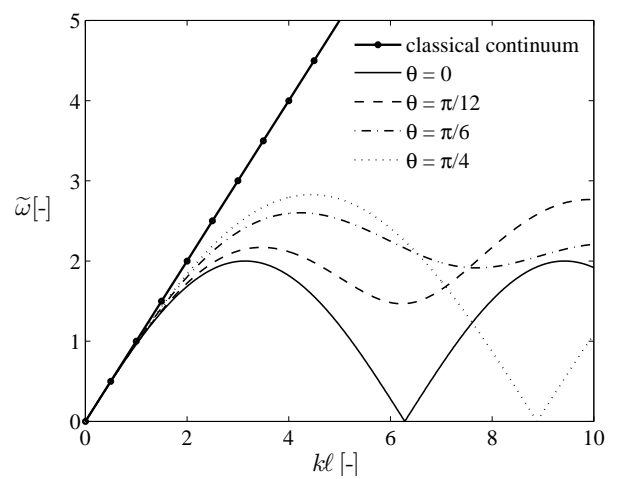

(b)

Figure 2. Dispersion curves for a square lattice and the classical continuum: (a) directional characteristics for wave propagating through the square lattice; (b) comparison of dispersion curves for the square lattice at different direction of propagation and the classical continuum approximation.

fixed $\omega$ and its magnitude is equal to the gradient of the dispersion surface. Hence, the direction of propagation of a wave at given frequency can be estimated by taking the normal to the iso-frequency contour lines (Langley 1997). This property in particular can be utilized to identify regions within the structure where waves at certain frequencies do not propagate in specified directions.

In $\S 2$ the discrete equation of motion has been transformed in a continuum model taking advantage of the continualisation approach. As previously illustrated, if in the series expansion only the terms up to the second derivatives are retained, the equation of motion yields the wave equation based upon a classical continuum model expressed by equation (2.6). Substituting into equation of motion the harmonic wave of the continuum form

$$
u(x, y ; t)=A \exp \left[\mathrm{i}\left(\omega t-k_{x} x-k_{y} y\right)\right]
$$

we find the solution

$$
\widetilde{\omega}=k \ell
$$

This equation shows that the classical continuum is not dispersive because the frequency $\omega$ is directly proportional to the wavenumber $k$.

The dispersion relations of the square lattice and the classical continuum model, as represented by equation (3.2) and equation (3.5), respectively, have been plotted in figure 2(b), which displays the behaviour of the dimensionless frequency $\widetilde{\omega}$ in terms of the normalized wave number $k \ell$. The dispersion curve for the continuum model is represented as a solid straight line through the origin, while the discontinuous lines represent the dispersion properties of the lattice for different angles $\theta$ in the range $\left[0, \frac{\pi}{4}\right]$. The comparison illustrates that the physical motion of the lattice system is well described by the classical continuum model for longer-wave limit. This means that when the wavelengths are much larger than the structural inhomogeneity of the lattice $(k \ell \rightarrow 0$ or $k \ell \ll 1)$, the waves are not affected by the heterogeneity of the membrane. 


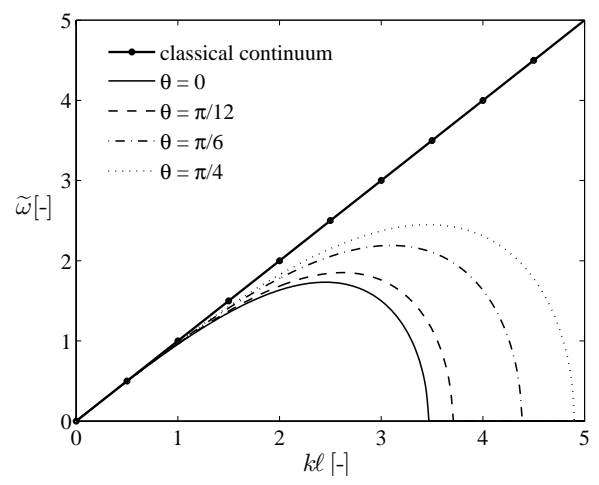

Figure 3. The $\widetilde{\omega}-k \ell$ dispersion curves for the fourth-order approximation of equation (3.7).

On the other hand, the dispersion becomes evident in the short-waves range, when the phase velocity $c_{p}$ of the harmonic waves, that is the speed at which the phase of any one component of the wave travels, is different from the group velocity $c_{g}$. The phase velocity $c_{p}$ can be expressed as

$$
c_{p}=\frac{\omega}{\sqrt{k_{x}^{2}+k_{y}^{2}}}
$$

and is graphically represented by the secant slope of the dispersion curves while the group velocity $c_{g}$ is represented by the tangential slope. By inspection of the curves the local continuum model appears unable to predict the decrease of phase velocity for increasing wavenumbers.

\section{(b) Dispersion relations for higher-order continua}

Retaining more terms in the series gives an enhanced continuum model represented by equation (2.10). Explicitly, we seek solutions of the harmonic form expressed by equation (3.4) that inserted into equation (2.10) give the relationship between the frequency $\omega$ and the wavenumber $k$, which in non-dimensional form turns out to be

$$
\widetilde{\omega}= \pm k \ell \sqrt{1-\frac{(k \ell)^{2}}{12}\left(1-\frac{\sin ^{2} 2 \theta}{2}\right)}
$$

From a mathematical point of view, the model governed by equation (3.7) produces wave dispersion if the function under the square root is greater than zero. When $k$ exceeds a critical value for fixed direction of propagation, the wave does not possess real frequency and the solution is unstable. The dispersion curves for the higherorder continuum, depicted in figure 3, show a downward branch crossing the axis $\widetilde{\omega}$ at values of $k \ell$ at which instability occurs. Because of the limitation due to the stability criterion, the applicability of equation (2.10) is restricted to the dynamic phenomena that do not involve short waves.

If asymptotic analysis is resorted to, a way to deal with the instability problem in the fourth-order continuum model suggests replacing the fourth-order derivative in equation (2.10) with mixed double time double space derivatives (Pichugin et al. 


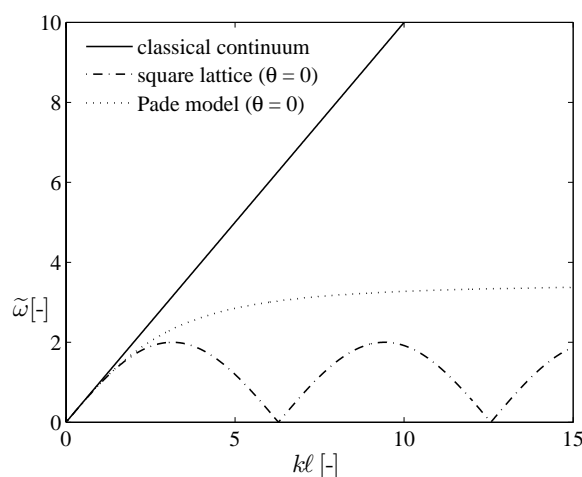

(a)

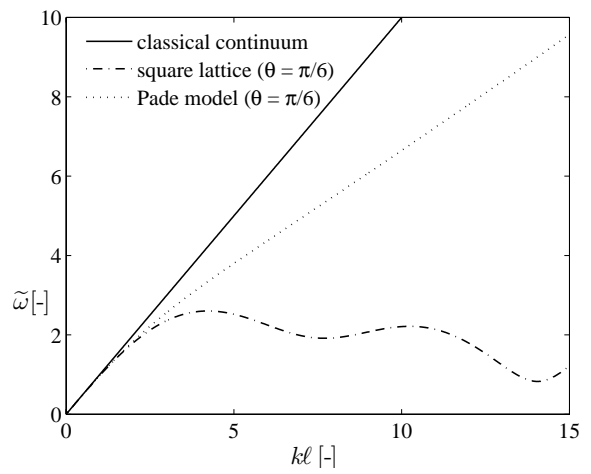

(c)

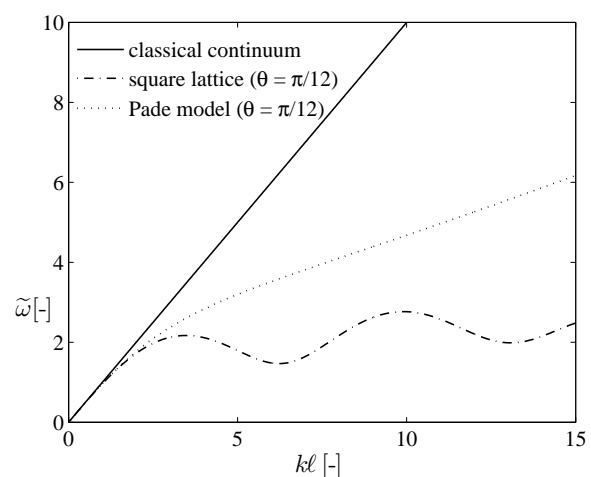

(b)

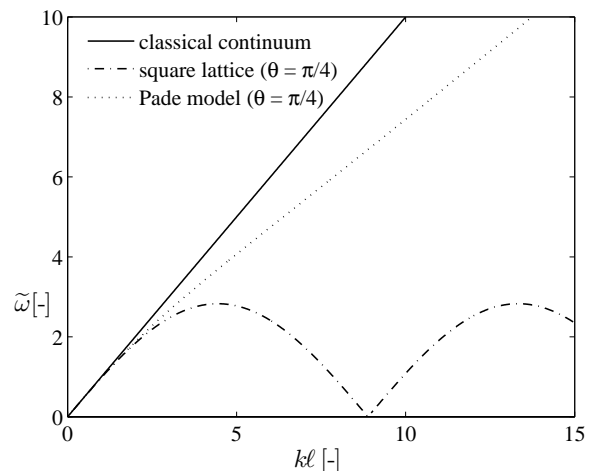

(d)

Figure 4. Dispersion curves for the enhanced continuum: (a) dispersion curves for different directions of propagation; (b),(c),(d) comparison of dispersion curves for the classical continuum, the square lattice and the Padé model for different directions of propagation.

2008). With the aim of restoring in the continuum approximation the dispersion property due to the discreteness of the square lattice whilst at the same avoiding instabilities, the Padé approximations can be employed. In the following, the model introduced in equation (2.12) is referred to as Padé continuum.

Solving the dispersion equation for the expected harmonic travelling wave, the following expression can be found

$$
\widetilde{\omega}= \pm k \ell \sqrt{\frac{1+\frac{(k \ell)^{2}}{24} \sin ^{2} 2 \theta}{1+\frac{(k \ell)^{2}}{12}}}
$$

It is worth remarking that the model is unconditionally stable since the frequencies defined by equation (3.8) are real in the complete range of wave numbers.

In figure 4 the dispersion curves for the Padé model are plotted in accordance with the relationships obtained for the square lattice and the classical continuum model for different directions of propagation. The plots show that at the long- 
range limit $k \ell \rightarrow 0$, the tangential slopes of the dispersion curves, corresponding to the group velocity $v_{g}=\frac{\partial \omega}{\partial k}$, are equal to those of the lattice model and the local continuum. For increasing wave numbers curves start to deviate from those of the discrete lattice model. However, they are located below the bold line that represents the local continuum model, and the group velocity is smaller than the one obtained for the local model, for all directions.

We note that the dispersion curve for the Padé continuum with $\theta=0$ tends to an horizontal asymptote. In this case, the slope (group velocity) for transverse wave is zero and frequencies above the limit value cannot propagate. Thus the model acts as a filter, where only relatively low frequencies are transmitted along the $\theta=0$ direction.

By considering its definition, the group velocity of waves for the Padé continuum can be found to depend on the wavenumber and the direction of propagation as

$$
c_{g}= \pm c \frac{288+(k \ell)^{2}\left(24+(k \ell)^{2}\right) \sin ^{2}(2 \theta)}{\sqrt{2}\left(12+(k \ell)^{2}\right) \sqrt{\frac{24+(k \ell)^{2} \sin ^{2}(2 \theta)}{12+(k \ell)^{2}}}}
$$

where $c=\sqrt{\frac{T}{\rho}}$ is the classical wave speed. This expression allow us to verify that propagating waves cannot transfer energy infinitely fast. In the long-wave limit, the group velocity takes the following form

$$
\lim _{k \rightarrow 0} c_{g}= \pm c
$$

and for short waves

$$
\lim _{k \rightarrow \infty} c_{g}= \pm c \frac{\sin ^{2}(2 \theta)}{\sqrt{2}}<c \quad \forall \theta
$$

Thus, the model governed by equation (2.12) is stable and provides lower and upper bounds for the speed of energy transfer by propagating waves (Metrikine 2006).

\section{Discretization of the equation of motion}

With the aim of finding approximate solutions of equation (2.12) governing the dynamics of the membrane structure modelled by using the enhanced continuum, in this section a spatial discretization based on the finite element method is carried out and the conditions for the convergence are reported. A weak form of the initial-boundary value problem is taken premultiplying equation (2.12) by a weight function $v$ and integrating over the domain $\Omega$, that is

$$
\int_{\Omega} v\left[\rho\left(1-\frac{\ell^{2}}{12} \nabla^{2}\right) \frac{\partial^{2} u}{\partial t^{2}}\right] \mathrm{d} \Omega=\int_{\Omega} v\left[T\left(\nabla^{2} u-\frac{\ell^{2}}{6} \frac{\partial^{4} u}{\partial x^{2} \partial y^{2}}\right)+p\right] \mathrm{d} \Omega
$$

The term $p$ represents the influence of a transverse applied force per unit area and it has been introduced in the last integral of equation (4.1) in order to generalize the initial-boundary value problem. Next, the divergence theorem is used to distribute the differentiation among $v$ and $u$, so the weak form of equation (2.12) is written 
as

$$
\begin{aligned}
& \int_{\Omega} v \rho \ddot{u} \mathrm{~d} \Omega+\int_{\Omega} \rho \frac{\ell^{2}}{12}\left(\frac{\partial v}{\partial x} \frac{\partial \ddot{u}}{\partial x}+\frac{\partial v}{\partial y} \frac{\partial \ddot{u}}{\partial y}\right) \mathrm{d} \Omega \\
&+\int_{\Omega} T\left(\frac{\partial v}{\partial x} \frac{\partial u}{\partial x}+\frac{\partial v}{\partial y} \frac{\partial u}{\partial y}\right) \mathrm{d} \Omega \\
&+\int_{\Omega} T \frac{\ell^{2}}{6} \frac{\partial^{2} v}{\partial x \partial y} \frac{\partial^{2} u}{\partial x \partial y} \mathrm{~d} \Omega=\int_{\Omega} v p \mathrm{~d} \Omega \\
&+\oint_{\Gamma} v T\left[n_{x}\left(1-\frac{\ell^{2}}{12} \frac{\partial^{2}}{\partial y^{2}}+\rho \frac{\ell^{2}}{12} \frac{\partial^{2}}{\partial t^{2}}\right) \frac{\partial u}{\partial x}+n_{y}\left(1-\frac{\ell^{2}}{12} \frac{\partial^{2}}{\partial x^{2}}+\rho \frac{\ell^{2}}{12} \frac{\partial^{2}}{\partial t^{2}}\right) \frac{\partial u}{\partial y}\right] \mathrm{d} \Gamma \\
&+\oint_{\Gamma} T \frac{\ell^{2}}{12}\left(\frac{\partial v}{\partial x} n_{y}+\frac{\partial v}{\partial y} n_{x}\right) \frac{\partial^{2} u}{\partial x \partial y} \mathrm{~d} \Gamma
\end{aligned}
$$

where $n_{x}$ and $n_{y}$ are the components of the unit normal vector on the boundary $\Gamma$ and a superimposed dot denotes the full derivative with respect to time. On the right side of the equation (4.2) the boundary integrals are collected whose contribution will be discussed afterwards in relation to the precise form of the boundary conditions of the problem. At this stage we assume homogeneous boundary conditions, so the boundary integrals cancel.

In deriving the finite element equations, the computational domain $\Omega$ is partitioned into bilinear isoparametric quadrilateral finite elements. This finite element is designated in the sequel as Q4 finite element (quadrilateral finite element with four nodes). The classical nodal interpolation procedure of the displacement field is assumed and the time dependence is separated from the spatial variation. The unknown displacement field $u(x, y, t)$ is approximated over a typical finite element $\Omega^{e}$ by using standard polynomial shape functions $N_{j}(j=1, \ldots, 4)$, as follows

$$
u(x, y, t)=\sum_{j} d_{j}^{e}(t) N_{j}^{e}(x, y)
$$

where $d_{j}, j=1, \ldots, 4$ are the nodal displacements of the $j$ th node of the element. The weight function $v$ is also discretized with the same shape functions. Because the weak form involves first-order and second-order mixed partial derivatives, $\mathscr{C}^{0}$ continuous shape functions can be used avoiding the cumbersome $\mathscr{C}^{1}$-continuity in the numerical implementation.

Substituting the finite element approximation in the weak form, the corresponding matrix form of equation (4.2) is given by

$$
\left[\boldsymbol{M}_{0}+\boldsymbol{M}_{2}\right] \ddot{\boldsymbol{d}}+\left[\boldsymbol{K}_{0}+\boldsymbol{K}_{2}\right] \boldsymbol{d}=\boldsymbol{P}
$$

with

$$
M_{i j}^{(0)}=\int_{\Omega^{e}} \rho N_{i}^{e} N_{j}^{e} \mathrm{~d} \Omega \quad K_{i j}^{(0)}=\int_{\Omega^{e}} T\left(\frac{\partial N_{i}^{e}}{\partial x} \frac{\partial N_{j}^{e}}{\partial x}+\frac{\partial N_{i}^{e}}{\partial y} \frac{\partial N_{j}^{e}}{\partial y}\right) \mathrm{d} \Omega
$$

are the classical elasticity contributions to the element mass and stiffness matrices, respectively, while

$$
\begin{aligned}
M_{i j}^{(2)} & =\int_{\Omega^{e}} \rho \frac{\ell^{2}}{12}\left(\frac{\partial N_{i}^{e}}{\partial x} \frac{\partial N_{j}^{e}}{\partial x}+\frac{\partial N_{i}^{e}}{\partial y} \frac{\partial N_{j}^{e}}{\partial y}\right) \mathrm{d} \Omega \\
K_{i j}^{(2)} & =\int_{\Omega^{e}} T \frac{\ell^{2}}{6} \frac{\partial^{2} N_{i}^{e}}{\partial x \partial y} \frac{\partial^{2} N_{j}^{e}}{\partial x \partial y} \mathrm{~d} \Omega
\end{aligned}
$$


are the adjunctive contributions to the element mass and stiffness matrices related to the enhanced continuum, and

$$
P_{i}=\int_{\Omega^{e}} N_{i}^{e} p \mathrm{~d} \Omega
$$

is the nodal force vector given by the externally applied pressure.

The equation of motion can be easily discretized and solved in the time domain by applying Newmark's time integration method. For an unconditionally stable solution, the constant-average acceleration scheme is used with no restriction on the time step.

\section{(a) Convergence and accuracy of the finite element solution}

The finite element method provides an approximate solution for a mathematical model associated with an actual physical problem. To proof the convergence of the numerical solution to the exact solution of the governing equations as the number of elements increases, a measurement for its quality is required. If an exact solution is not available, the convergence can be measured only on the fact that some conditions contained in the mathematical model must be ultimately (at convergence) satisfied (Reddy 1993).

The accuracy of the FEM for the membrane problem when the enhanced continuum model is used may be assessed, for instance, from the convergence of the strain energy. The strain energy actually contained in the finite element model can be evaluated via

$$
U=\frac{1}{2} \boldsymbol{d}^{T}\left[\boldsymbol{K}_{0}+\boldsymbol{K}_{2}\right] \boldsymbol{d}
$$

In order to illustrate the performance of the Q4 membrane elements, the convergence in strain energy is shown in figures $5(\mathrm{a})$ and $5(\mathrm{~b})$ for two sequences of uniform and skewed meshes obtained halving the element size for the static problem of a membrane simply supported on the boundary and loaded by a uniform pressure. The plots give an error estimate if the convergence of strain energy has been achieved. In fact, as both meshes are refined, the strain energy approaches the exact solution from below.

Another, more frequently used, approach of quantifying the discretization error is the relative energy norm defined as follows

$$
e=\sqrt{\frac{U-U_{r e f}}{U_{r e f}}}
$$

where the reference energy $U_{\text {ref }}$ corresponds to the finest mesh. This measure of error is closely related to the error on the derivative of the computed displacement field.

Error estimates of the type equation (4.9) are very useful because they give qualitative information on the accuracy of the approximate solution, whether or not the true solution is known. The energy norm as a function of mesh refinement is reported in figures 5(c) and 5(d) in logarithmic scaling such that an algebraic curve is mapped onto a straight line. It can be clearly seen that the error decreases with the mesh refinement. 


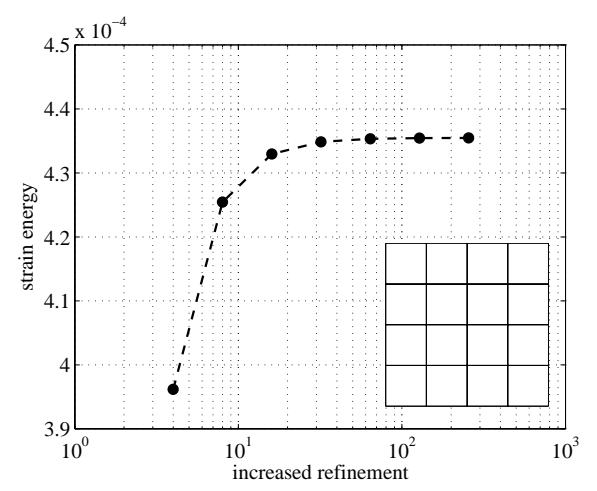

(a)

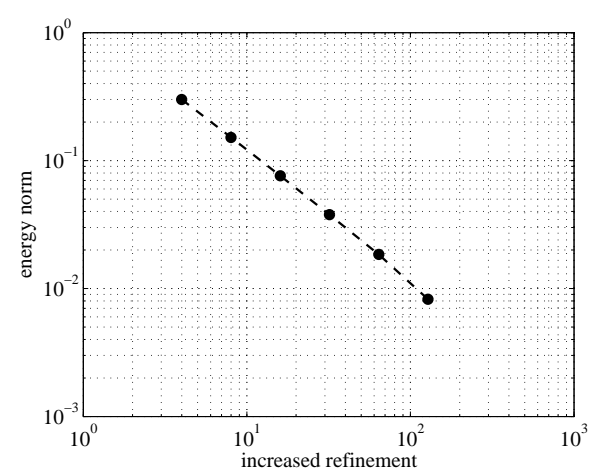

(c)

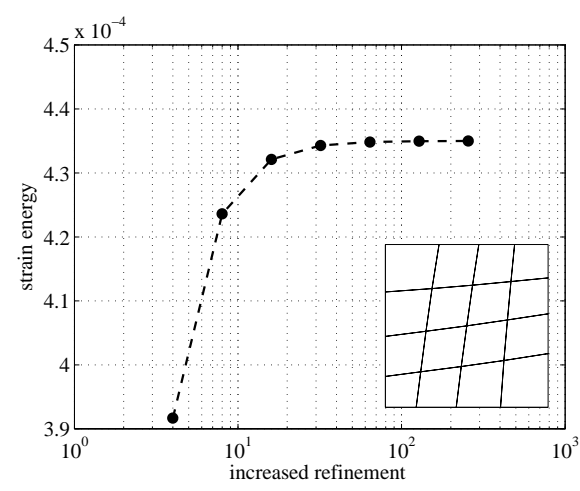

(b)

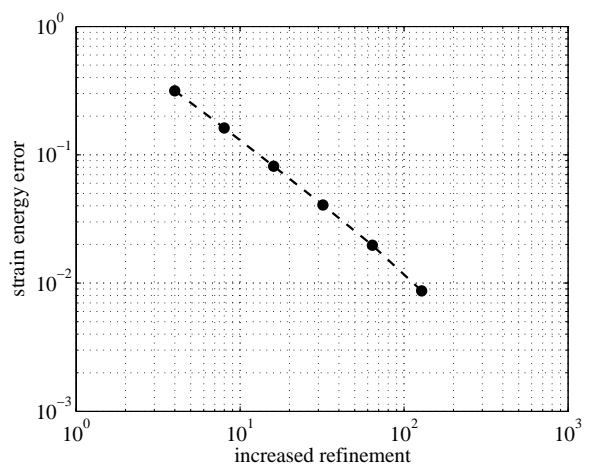

(d)

Figure 5. Convergence of the numerical energy and convergence rate for a sequence of meshes with regular $(\mathrm{a}, \mathrm{c})$ and quasi-regular $(\mathrm{b}, \mathrm{d})$ patterns.

The error in the energy norm is proportional to $h$ that represents a typical length of an element side and it is expressed as

$$
e=C h^{-(k+1-m)}
$$

where $k$ is the degree at which the interpolant polynomial is complete and $m$ is the order of the highest derivative of the displacement field in the weak form (Zienkiewicz \& Taylor 2000, Akin 2005). The element investigated here uses a polynomial displacement complete to the degree $k=1$ and in the weak form the highest derivative order is of first order in the spatial dimensions $(m=1)$. Thus, a rate of convergence $p=k+1-m=1$ is expected. As can be observed from figures 5 (c) and $5(\mathrm{~d})$ the convergence obtained in the numerical simulation compares very well with the theoretical value.

Finally, having compared the numerical results obtained for the two test meshes, it is evident that the orders of convergence are not affected by the element distortions. 


\section{Numerical examples}

In $\S 2$, the equation of motion of a microstructured membrane has been derived by means of non-local continuum mechanics models and in $\S 4$ the discretisation of the equation in the spatial and temporal domain is presented. Based on the formulations obtained above, to examine the microstructure effects on the dynamic response of a membrane simply supported on the edges, the results obtained from classical and higher-order elasticity (Padé continuum) are discussed in this section.

The geometry and the boundary conditions of the squared microstructured membrane are given on the left of figure 6 . The length and the width of the panel is $2 L=2 \mathrm{~m}$, the material density is $\rho=1 \frac{\mathrm{kg}}{\mathrm{m}^{2}}$. The parameter $\ell$ is the microstructural characteristic length. The restoring force in the vibrating membrane arises from the tension $T$ at which it is stretched and the in-plane tension $T=16 \frac{\mathrm{N}}{\mathrm{m}}$ is chosen for the current analysis.
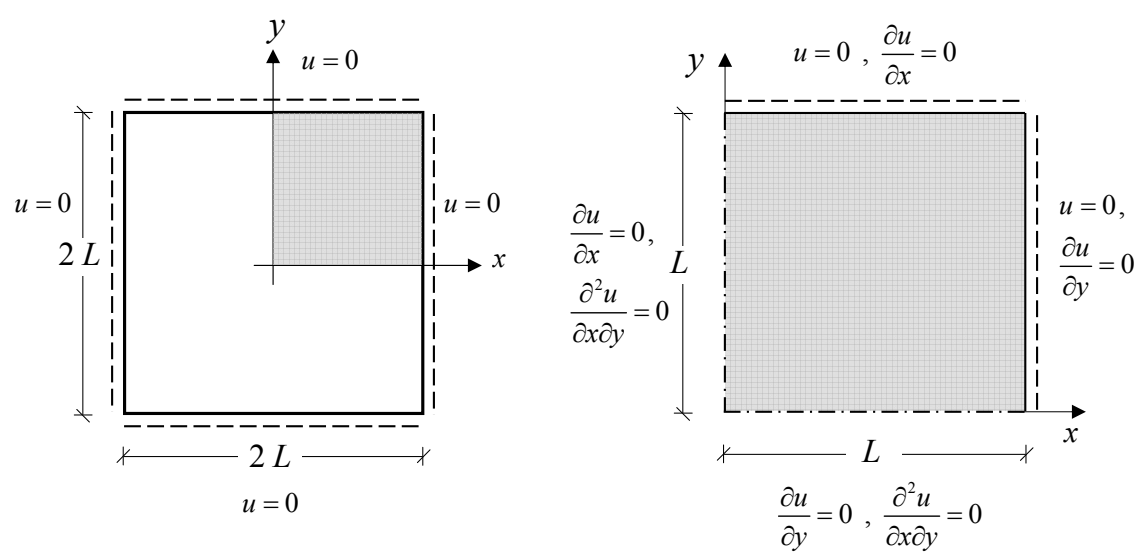

Figure 6. Geometry and boundary conditions of the membrane problem and top-right quarter of the membrane and boundary conditions.

Because the dynamical response of a structure is mainly dependent on the dynamic load applied and on the dynamic characteristic of the structure itself, the analysis of natural frequencies is an important topic. The modal properties of a membrane can be obtained solving the standard matrix eigenproblem

$$
\left[-\omega^{2} \boldsymbol{M}+\boldsymbol{K}\right] \boldsymbol{d}=\mathbf{0}
$$

where $\omega$ is the finite element predicted natural frequency of the system and $\boldsymbol{d}$ is the corresponding natural mode of the system.

Since analytical solutions are available just for the classical continuum model and a mesh refinement allows to obtain more accurate results, a convergence analysis has been carried out in order to assess how many Q4 elements should be used in the numerical calculations. The frequencies based on classic elasticity and higherorder models are given in figure 7 . The results associated with $\ell=0$ correspond to those of the classical elasticity theory where the microstructural effect is ignored. It can be seen that the difference of the frequencies for different values of $\ell$ becomes 


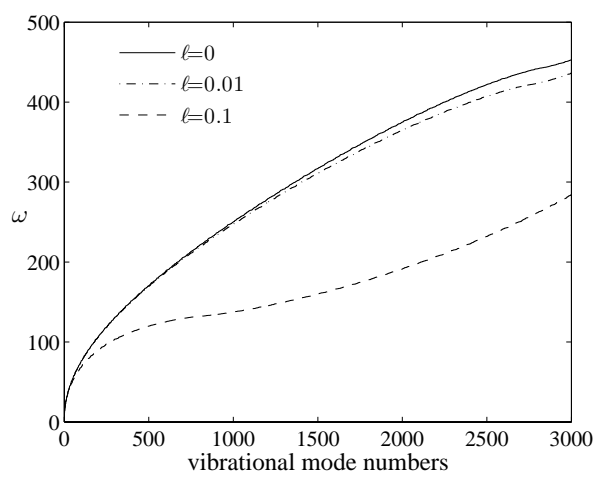

Figure 7. Comparison of natural frequencies computed using classical and higher-order continuum models.

evident at higher vibrational modes, although this difference is negligible at lower vibrational mode numbers. Further, the frequency decreases when the characteristic internal length $\ell$ increases. Hence, the frequencies are always overestimated by the classical continuum model.

The second numerical application deals with the transient response of the microstructured membrane subjected at its center to an impact excitation. The dynamic load has been modelled as an initial non-zero velocity $v\left(t_{0}\right)=1 \frac{\mathrm{m}}{\mathrm{s}}$ at the impact point. Due to the biaxial symmetry of the problem, we use a uniform mesh of Q4 elements to discretize only the top-right quadrant of the domain. The axes of symmetry $x=0$ and $y=0$ become a portion of the boundary $\Gamma$ of the computational domain and the kinematic considerations reported in figure 6 allow us to justify the assumption of homogeneous boundary conditions introduced in $\S 4$. The Newmark scheme is used for the time integration of the equations using the recommendations on time step size proposed by Bennett and Askes, 2009.

Figures $8(\mathrm{a})$ and $8(\mathrm{~b})$ show the wave fronts at times $t=0.10,0.20$ and $0.25 \mathrm{~s}$ for classical elasticity, obtained taking $\ell=0$, and the Padé model for microstructural length $\ell=0.01$, respectively. As previously predicted in $\S 3$ by means of the dispersion analysis, the elastic waves obtained using the local elasticity theory propagate faster than the case of the enhanced continua. The wave fronts for both models are governed by the components with higher wavelengths (small $k$ ) because they travel faster than the shorter ones. In fact, the velocity of the long waves is not influenced by the direction of propagation and the fronts have quite a circular shape. The directional properties of the microstructured membrane expected for the enhanced continuum model can be observed from figure 8 (b) focusing the attention on the short waves that propagate slower and whose velocities vary with the direction of propagation. The directional property of the Padé model can be better observed in figures 9 (a) and 9 (b) where elastic waves propagating from the excitation point to the boundary for different characteristic lengths and along two specific directions are plotted at time $t=0.20 \mathrm{~s}$. 

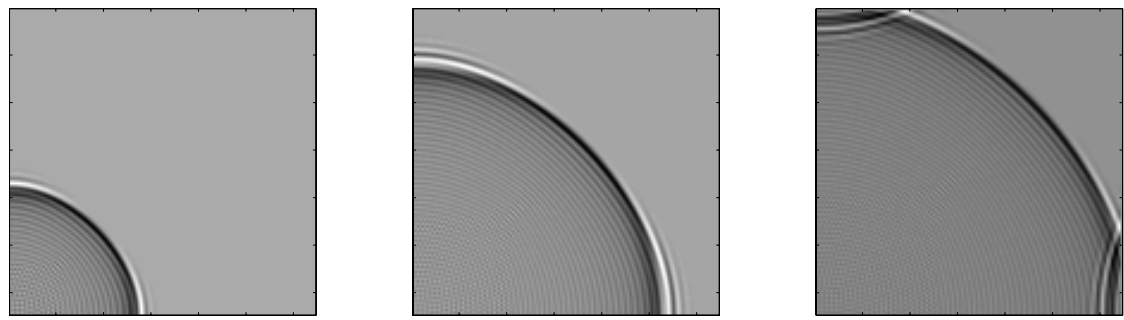

(a)
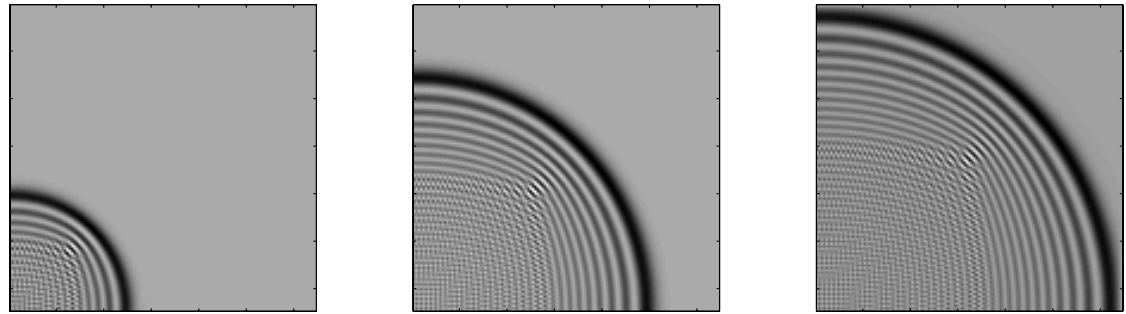

(b)

Figure 8. Fronts of propagating elastic waves in microstructured membrane: (a) classical continuum model; (b) enhanced Padé continuum model.

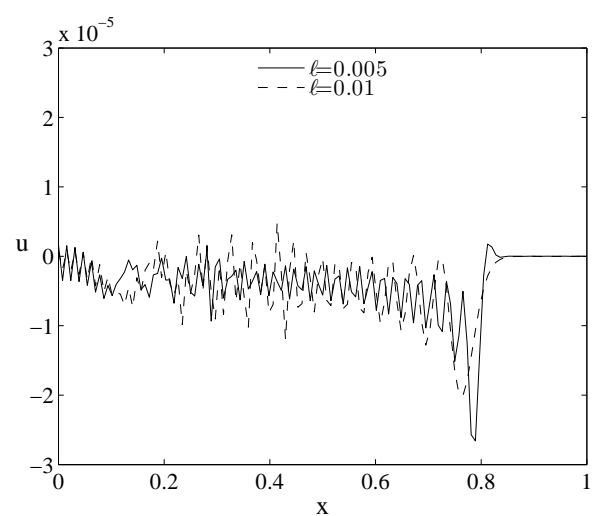

(a)

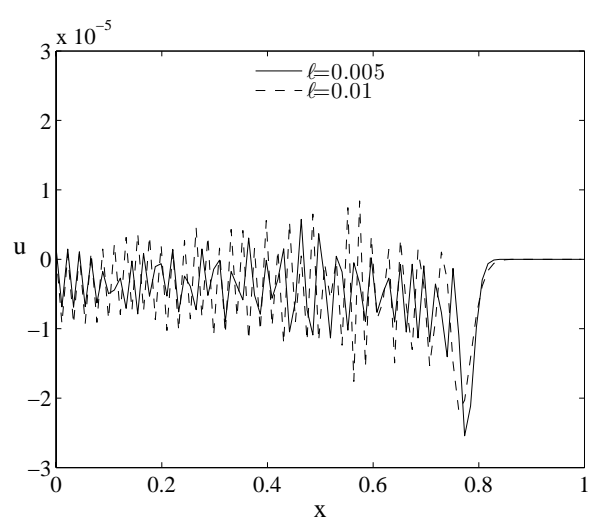

(b)

Figure 9. Propagating waves for enhanced continuum model (Padé model) and two microstructural lengths $\ell$ : (a) direction $\theta=0$; (b) direction $\theta=\frac{\pi}{4}$.

\section{Conclusions}

This contribution addresses the problem of wave dispersion in composite membranes that possess a microscopic structure. Among other observed phenomena that are influenced by the microstructural characteristics, the dispersion of waves propagating in composites is of great interest in the mechanics of materials because it plays a crucial role for instance in stopping the formation of shock waves into 
systems. As an alternative to the direct analysis of the discrete material that at microscopic level has been assumed here as a periodic elastic lattice, a continuum model approximating the actual material behaviour has been employed. With the aim to take into account in the dynamics the dispersion of the wave born out of heterogeneity of the material, a higher-order theory has been successfully resorted to, overcoming the limits of classical elasticity in the high-frequency regime.

In the first part of this study, continuum models linked with the underlying material have been introduced taking into account the discreteness of the microstructure. From the application of different continualisation approaches, it has been shown that the higher-order theories improve the continuum description by including the microstructural effects. Specifically, enhanced continuum theories based on the use of Padé approximants introduce higher-order inertia and stiffness terms in classical models and improve the continuum description by including the length parameter characterizing the heterogeneity. The analysis of closed-form dispersion relations demonstrated that the enhanced continuum models deliver physically consistent results also in the limit of short waves and do not generate numerical instability.

Further, the field equations have been discretized in space by means of simple four noded finite elements. In the implementation the $\mathscr{C}^{1}$-continuity is completely avoided. In fact, the weak form of the governing equations involves first-order and second-order mixed derivatives of the unknown field, thus $\mathscr{C}^{0}$ continuous shape functions are sufficient. Importantly, the higher-order terms do not lead to an increase in system size. The evaluation of the convergence rate confirms the appropriateness of the adopted finite element discretisation.

Finally, the analysis of a microstructured membrane has been conducted. The vibration frequencies have been estimated solving the standard matrix eigenproblem for the classical and enhanced continuum models. It has been found that the frequencies are always overestimated by the classical continuum model. In order to illustrate the dispersion of the elastic waves, the response of the membrane to impulsive point load has been simulated. Numerical results are in agreement with the analytical dispersion analysis presented in this paper.

\section{Acknowledgments}

We gratefully acknowledge the financial support of the Engineering and Physical Sciences Research Council, contract number EP/D041368/1.

\section{References}

Akin, J. E. 2005 Finite element analysis with error estimators, Elsevier.

Andrianov, I. V. \& Awrejcewicz, J. 2005 Continuous models for 1D discrete media valid for higher-frequency domain. Phys. Lett. A 345, 55-62.

Andrianov, I. V. \& Awrejcewicz, J. 2008 Continuous models for 2D discrete media valid for higher-frequency domain. Comput. Struct. 86, 140-144.

Bennett, T. \& Askes,A., J. 2009 Finite element modelling of wave dispersion with dinamically consistent gradient elasticity. Comput. Mech. 43, 815-825.

Chang, C. S. \& Gao, J. 1995 Second-gradient constitutive theory for granular material with random packing structure. Int. J. Solids Struct. 32, 2279-2293. 
Chen, W. \& Fish, J. 2001 A dispersive model for wave propagation in periodic heterogeneous media based on homogenization with multiple spatial and temporal scales. $J$. Appl. Mech.-T. ASME 68, 153-161.

Erofeyev, V. I. 2003 Wave processes in solids with microstructure, World Scientific, Singapore.

Fish, J., Chen, W. \& Nagai, G. 2002 Non-local dispersive model for wave propagation in periodic heterogeneous media: one-dimesional case. Int. J. Numer. Meth. Eng. 54, $331-346$.

Jakata, K. \& Every, A. G. 2008 Determination of the dispersive elastic constants of the cubic crystals Ge, Si, GaAs, and InSb. Phys. Rev. B 77, 1-10.

Jenkins, C. H. M. \& Korde, U. A. 2006 Membrane vibration experiments: An historical review and recent results. J. Sound Vib. 295, 602-613.

Kevrekidis, P. G., Kevrekidis, I. G., Bishop, A. R. \& Titi, E. S. 2002 Continuum approach to discreteness. Phys. Rev. E 65, 1-13.

Langley, R.S. 1997 The response of two-dimensional periodic structures to impulsive point loading. J. Sound Vib. 201, 235-253.

Metrikine, A. V. \& Askes, H. 2002 One-dimensional dynamically consitent gradient elasticity models derived from a discrete microstructure Part 1: Generic formulation. Eur. J. Mech. A: Solids 21, 555-572.

Metrikine, A. V. 2006 On causality of the gradient elasticity models. J. Sound Vib. 297, $727-742$.

Mühlhaus, H.-B. \& Oka, F. 1996 Dispersion and wave propagation in discrete and continuous models for granular materials. Int. J. Solids Struct. 33, 2841-2858.

Papargyri-Beskou, S., Polyzos, D. \& Beskos, D. E. 2009 Wave propagation in gradient elastic solids and structures: A unified treatment. Int. J. Solids Struct. 46, 3751-3759.

Pichugin, A. V., Askes, A. \& Tyas, A. 2008 Asymptotic equivalence of homogenization procedures and fine-tuning of continuum-theories. J. Sound Vib. 313, 858-874.

Reddy, J. N. 1993 An introduction to finite element method, 2nd edn. McGraw-Hill.

Rosenau, P. 1986 Dynamics of nonlinear mass spring chains near the continuum limit. Phys. Lett. A 118, 222-227.

Rosenau, P. 1987 Dinamics of dense lattices. Phys. Rev. B 36, 5868-5876.

Suiker, A. S. J., Metrikine, A. V. \& de Borst, R. 2001 Comparison of wave propagation characteristics of the Cosserat continuum model and corresponding discrete lattice models. Int. J. Solids Struct. 38, 1563-1583.

Suiker, A. S. J. \& de Borst, R. 2005 Enhanced continua and discrete lattices for modelling granular assemblies. Proc. R. Soc. Lond. A 363, 2543-2580.

Wattis, J. A. D. 2000 Quasi-continuum approximations to lattice equations arising from the discrete nonlinear telegraph equation. J. Phys. A 33, 5925-5944.

Zienkiewicz, O. C. \& Taylor, R. L. 2000 The finite element method: The basis, 5th edn. Butterworth-Heinemann. 


\section{List of figures}

1. Two-dimensional discrete lattice in the $(x, y)$ plane.

2. Dispersion curves for a square lattice and the classical continuum: (a) directional characteristics for wave propagating through the square lattice; (b) comparison of dispersion curves for the square lattice at different direction of propagation and the classical continuum approximation.

3 . The $\widetilde{\omega}-k \ell$ dispersion curves for the fourth-order approximation of equation (3.7).

4. Dispersion curves for the enhanced continuum: (a) dispersion curves for different directions of propagation; (b), (c),(d) comparison of dispersion curves for the classical continuum, the square lattice and the Padé model for different directions of propagation.

5. Convergence of the numerical energy and convergence rate for a sequence of meshes with regular $(\mathrm{a}, \mathrm{c})$ and quasi-regular $(\mathrm{b}, \mathrm{d})$ patterns.

6. Geometry and boundary conditions of the membrane problem and top-right quarter of the membrane and boundary conditions.

7. Comparison of natural frequencies computed using classical and higher-order continuum models.

8. Fronts of propagating elastic waves in microstructured membrane: (a) classical continuum model; (b) enhanced Padé continuum model.

9. Propagating waves for enhanced continuum model (Padé model) and two microstructural lengths $\ell$ : (a) direction $\theta=0$; (b) direction $\theta=\frac{\pi}{4}$. 